\title{
In situ delivery of thermosensitive gel-mediated 5-fluorouracil microemulsion for the treatment of colorectal cancer
}

This article was published in the following Dove Press journal:

Drug Design, Development and Therapy

8 September 2016

Number of times this article has been viewed

\section{Lu-Lu Wang \\ Shuai Huang \\ Hui-Hui Guo \\ Yan-Xing Han \\ Wen-Sheng Zheng \\ Jian-Dong Jiang}

State Key Laboratory of Bioactive Substance and Function of Natural Medicines, Institute of Materia Medica, Chinese Academy of Medical Sciences and Peking Union Medical College, Beijing, People's Republic of China
Correspondence: Yan-Xing Han; Jian-Dong Jiang Institute of Materia Medica, Chinese Academy of Medical Sciences and Peking Union Medical College, I Xian Nong Tan Street, Beijing 100050, People's Republic of China

Tel +86 10 63165233

Fax +861063017757

Email hanyanxing@imm.ac.cn; jiang.jdong@163.com

\begin{abstract}
In situ administration of 5-fluorouracil (5FU) "thermosensitive" gel effectively reduced systemic side effects in treating colon rectal cancer; however, the penetration efficacy of the formulation was considerably low due to the poor lipid solubility of 5FU. The aim of this study was to develop thermosensitive gel-mediated 5FU water-in-oil microemulsion (TG-5FU-ME) for improving the infiltration of 5FU. An in vitro release test showed that TG-5FU-ME sustained the drug's release up to 10 hours. TG-5FU-ME exhibited good stability, and the microemulsion entrapped did not show any change in morphology and 5FU content during the 4-month storage. Transportation test in the Caco-2 cell monolayer showed that TG-5FU-ME had a permeability 6.3 times higher than that of 5FU thermosensitive gel, and the intracellular uptake of 5FU increased by 5.4-fold compared to that of 5FU thermosensitive gel. In vivo tissue distribution analysis exhibited that the TG-5FU-ME group had drug levels in rectal tissue and mesenteric lymph nodes, which were significantly higher than those of 5FU thermosensitive gel group, with very low blood levels of 5FU in both groups. Furthermore, TG-5FU-ME was not associated with detectable morphological damage to the rectal tissue. Conclusively, TG-5FU-ME might be an efficient rectal delivery system to treat colorectal cancer.
\end{abstract}

Keywords: 5-fluorouracil, microemulsion, thermosensitive gel, Caco-2 transport, rectal delivery, colorectal cancer

\section{Introduction}

Colorectal cancer is the third most commonly diagnosed cancer in males and the second in females, ${ }^{1}$ with more than 1.4 million new cases and 694,000 deaths in $2012 .{ }^{2}$ It was estimated that colorectal cancer is one of the four most frequently diagnosed types of cancer and continued to be the most common causes of cancer death in $2014 .^{3}$ 5-Fluorouracil (5FU) is one of the main chemotherapeutic agents used in the clinic for the treatment of colon rectal cancer. ${ }^{4}$ Due to its low lipophilicity $(\log P,-0.83), 5 \mathrm{FU}$ shows poor oral bioavailability. ${ }^{5,6}$ For intravenous administration, a high dose of $5 \mathrm{FU}$ is required because of its rapid clearance from plasma (a mean half-life of $\sim 16$ minutes). ${ }^{7,8}$ High dose of $5 \mathrm{FU}$ is often accompanied by severe toxic effects in gastrointestinal, hematological, neural, cardiac, or dermatological systems. ${ }^{9,10}$ Intravenous administration of 5FU also causes physical and psychic pain, as well as hypertrophy or atrophy at the subcutaneous injection site. Therefore, finding an alternative delivery form is desirable in hoping to improve the current chemothrapeutic regimen. ${ }^{11}$

Delivering 5FU directly to the rectal site is considered a promising approach to reduce systemic side effects and provide effective tumor diffusion. ${ }^{12,13}$ Numerous studies have shown that the colon suppository administration of 5FU appeared to be efficient 
and showed pharmacokinetics similar to that of intravenous administration, but with decreased systemic side effects and increased rectal tissue drug concentration. ${ }^{14-19}$ Some animal studies demonstrated that administration of 5FU emulsion in the rectal mucous improved drug accumulation in regional lymph nodes, suggesting that 5FU emulsion therapy might be of potential in treating lymph node metastasis from rectal cancer. ${ }^{20,21}$ However, the solid form, ie, suppositories, was confined to limited drug contact area with the lesion and poor drug permeability crossing rectal mucous membrane. Other disadvantages of 5FU suppositories include anal pain, tenesmus, anal bleeding, reddened mucous, and histological changes coinciding with acute colitis. ${ }^{15,22}$ In addition, rectal administration of liquid forms often causes anal leakage, leading to inadequate dosing. ${ }^{13}$

The aim of this study was to develop an effective rectal delivery system of 5FU by simultaneously employing nanotechnology and thermosensitive gel technique, ${ }^{23-25}$ in which the microemulsion might improve drug permeation, sustain drug release, and reduce drug mucosal irritation. ${ }^{26-29}$ We assume that loading the 5FU microemulsion in the thermosensitive gel might improve its stability, tissue deposition, and patient compliance. ${ }^{30-33}$ To the best of our knowledge, this is the first attempt to combine the two technologies for rectal drug delivery. Indeed, thermosensitive gel-mediated 5FU water-in-oil microemulsion (TG-5FU-ME) sustained the drug release, increased the drug permeation, improved the drug stability, elevated the drug deposition in disease tissue, and meliorated the use convenience. Animal experiments exhibited that TG-5FU-ME group had significantly high drug levels in the rectal tissue and mesenteric lymph nodes, while it had low drug levels in the blood system with no detectable morphological damage to rectal tissue. The results suggest that TG-5FU-ME is a promising rectal drug delivery system in treating rectal cancer.

\section{Materials and methods Materials}

5FU was purchased from Xing Galaxy Chemical Company (Hubei, People's Republic of China). Poloxamers were gifted from BASF (Ludwigshafen, Germany), and sodium alginate was supplied by Huzhou Zhanwang Pharmaceutical Co., Ltd (Zhejiang, People's Republic of China). Tween 80 and Span 80 were supplied by Huamei Co., Ltd (Beijing, People's Republic of China). Soybean oil was supplied by Sigma-Aldrich Co. (St Louis, MO, USA). All other reagents were at analytical grade and used without further purification.

\section{Methods}

\section{Preparation and characterization of 5FU} microemulsion

Preparation of 5FU microemulsion

As described in previous reports, ${ }^{26,27,29}$ 5FU water-in-oil microemulsion was prepared by gradually adding $33 \mathrm{~mL}$ of drug-water solution into $33 \mathrm{~mL}$ oil phase containing liquid soybean oil, Span 80, Tween 80, and propylene glycol (21:5:1:6) under continuous stirring at 2,000 rpm for 10 minutes at $40^{\circ} \mathrm{C}$.

\section{Characterization of $5 \mathrm{FU}$ microemulsion}

Morphology test: A drop of drug-loaded sample was placed on a copper grid, and the excess was removed with filter paper. Phosphotungstic acid was applied for several minutes as a negative stain. The solution that overflowed was cleared and dried in the air. The grid was examined under a transmission electron microscope (Hitachi H-7650; Hitachi Ltd., Tokyo, Japan).

Particle size, polydispersity index, viscosity, and zeta potential test: The viscosity of each of the drug-loaded sample was tested using a DV-E Viscometer (Brookfield Engineering Laboratories, Inc., Middleboro, MA, USA), and the particle size, polydispersity index, and zeta potential of each of the drug-loaded sample were estimated using a Mastersizer (NICOMPтм 380 ZLS; Malvern, Santa Barbara, CA, USA) after dilution with filtered oil. The samples were diluted five times to keep the intensity between $300 \mathrm{kHz}$ and $350 \mathrm{kHz}$, and the scattering angle was 173 .

Entrapment efficiency test: Entrapment efficiency was determined by dialysis. Drug-loaded sample was added to a dialysis bag MD34 (MWCO 7,000; Viskase Co., Darien, IL, USA) and dialyzed for 24 hours at $4^{\circ} \mathrm{C}$. The concentrations of the drugs in the dialysis fluid were analyzed using high-performance liquid chromatography (HPLC) with the mobile phase of acetonitrile-water (95:5, $\mathrm{pH} 3.5)$ and a flow rate of $1 \mathrm{~mL} \mathrm{m^{-1 }}$. The standard curve, $A=59.957 X+10.373$ $(r=0.9992)$, was plotted with a peak area of $5 \mathrm{FU}$ as ordinate against its concentration as abscissa. The linear range was 0.1-20 $\mu \mathrm{g} \mathrm{mL}^{-1}$; the recovery rate was $99.2 \%$; the relative standard deviation (RSD) detected at 0 hour, 4 hours, 12 hours, and 24 hours was $2.1 \%$; and the RSD of six repetitions was $2.2 \%$ by detecting $5 \mathrm{FU}$ peak area. The entrapment efficiency was calculated as follows:

$$
\mathrm{EE}=100-C
$$

where $C$ is the amount of drug in the dialysis fluid expressed as a percentage. 


\section{Preparation and physicochemical properties of TG-5FU-ME}

\section{Preparation of TG-5FU-ME}

Appropriate quantities of $\mathrm{P} 407, \mathrm{P} 188$, and sodium alginate were added to the deionized water and mixed. The liquid was placed at $4{ }^{\circ} \mathrm{C}$ until a clear solution was obtained. The microemulsion formulation was mixed with thermosensitive gel under mechanical stirring at $4^{\circ} \mathrm{C}$ for 30 minutes at $700 \mathrm{rpm}$ followed by increasing the temperature slowly to ambient temperature.

\section{Physicochemical properties of TG-5FU-ME}

Measurement of gelation temperature: The measurement of gelation temperature was detected as described earlier. ${ }^{28-32}$ In brief, a $20 \mathrm{~mL}$ transparent beaker containing a magnetic bar and $10 \mathrm{~g}$ liquid gel was placed in a low-temperature thermostat water bath. The liquid gel was then heated at a constant rate while stirring. When the stirring magnet was arrested due to gelation, the temperature displayed on the thermistor was identified as the gelation temperature.

Measurement of gel strength: As described by Klimaszewsk, et al., ${ }^{34}$ CT3 Texture Analyzer (Brookfield Engineering Laboratories, Inc.) was used to test the gel strength. Samples were prepared in $25 \times 150 \mathrm{~mm}$ culture tubes until the liquid almost reached the top of the tube and equilibrated at $25^{\circ} \mathrm{C} \pm 1^{\circ} \mathrm{C}$ for 24 hours. The gel strength was tested with a $4 \mathrm{~mm}$-diameter stainless steel cylinder probe, with the trigger force fixed at $4 \mathrm{~g}$, the test distance depth at $2 \mathrm{~mm}$, and the test speed at $1 \mathrm{~mm} \mathrm{~s}^{-1}$. The gel strength was represented as the maximum peak force. For each sample, three replicates from different tubes were measured.

Measurement of coverage area: The coverage area was measured using the method described in our previous report. ${ }^{13}$ In brief, a section of tissue was taken from the rabbit rectum and secured with the mucosal side facing out on a glass plate and storing at $36.5^{\circ} \mathrm{C}$. Liquid gel $(1 \mathrm{~mL})$ was administered to the surface of the rabbit rectum, spreading freely until a gel film formed, and the area of the gel coverage was calculated.

Measurement of bioadhesive force: The bioadhesive force was determined using the method described by Koffi et $\mathrm{al}^{35}$ with little modification. Briefly, the ample gel was placed in contact with a fragment of rabbit rectal mucosa of certain surface area under thermostated conditions, and then adhesive joint between the two surfaces was formed. The gel strength was recorded as the maximal detachment force. A mucosal fragment (the surface area was determined by the measurement of coverage described earlier) was placed at the surface of a metallic plot with a rubber band, and this metallic plot was fastened to the mobile traverse of CT3 Texture Analyzer. A hollow plastic support (10 mm in depth) was filled with a preset amount of $3 \mathrm{~g}$ of the sample and was immobilized in a beaker filled with $300 \mathrm{~mL}$ of water $\left(37^{\circ} \mathrm{C}\right)$, which was immobilized in the texture analyzer apparatus. The whole system was placed in such a position that a perfect contact could be created between the surface of the gel and the mucous membrane. After being contacted for 10 minutes under an initial contact strength $(0.5 \mathrm{~N})$, the two surfaces were separated at a constant rate of displacement. The strength was recorded as the maximal detachment force, $F_{\text {max }}$.

\section{Formulation stability test}

Three batches of 5FU microemulsion and TG-5FU-ME were stored in sealed glass containers under the temperature of $20^{\circ} \mathrm{C} \pm 1{ }^{\circ} \mathrm{C}$ for 4 months. The stability was detected periodically by visual appearance, microscopy, and $5 \mathrm{FU}$ content.

The appearances of 5FU microemulsion and TG-5FU-ME were examined for layering, creaming, coalescence, phase separation, or precipitation. The $5 \mathrm{FU}$ microemulsion samples were examined at 0 hour, 24 hours, 72 hours, and 1 week, and TG-5FU-ME samples were tested in fresh as well as at 1 month, 2 months, 3 months, and 4 months after preparation. Each visual examination was accompanied with microscopic test for droplet structure change, merging, or rupturing using the OLYMPUS-B201/IX2-sp (1,000×; Olympus, Tokyo, Japan). The drug content of 5FU microemulsion or TG-5FU-ME was extracted by $2 \mathrm{~mol} \mathrm{~L}^{-1}$ hydrochloride and analyzed by HPLC (as discussed in the "Entrapment efficiency test" section).

\section{In vitro drug release}

The in vitro release of 5FU was determined by dynamic dialysis. A certain amount of $5 \mathrm{FU}$ microemulsion or TG-5FU-ME was transferred to prepare dialysis channels, which were placed in a conical flask filled with $100 \mathrm{~mL}$ of phosphate buffer solution ( $\mathrm{pH} 8.0)$ and magnetically stirred at $37^{\circ} \mathrm{C}$ at $150 \mathrm{rpm}$. Two milliliter sample was taken at 0.5 hours, 1 hour, 1.5 hours, 2 hours, 3 hours, 4 hours, 6 hours, 8 hours, 10 hours, 12 hours, and 14 hours, and the same volume of phosphate buffer solution was added into the flask simultaneously. The concentration of dialyzed drug was determined using HPLC, as mentioned earlier, to calculate the cumulative dissolution. The control samples for the experiment were the 5FU in water solution as well as the 5FU thermosensitive gel. The blank microemulsion and gel presented in the experimental system had no effect on the 5FU content measurement. 


\section{Trans Caco-2 cell test}

As described earlier, ${ }^{34,35}$ Caco-2 cell lines obtained from the Institute of Biochemistry and Cell Biology (Shanghai, People's Republic of China) were grown at $37^{\circ} \mathrm{C}$ in an atmosphere of $5 \% \mathrm{CO}_{2}$ and $90 \%$ relative humidity in $25 \mathrm{~cm}^{2}$ culture dish with standard growth media of Dulbecco's Modified Eagle's Medium supplemented with 10\% fetal bovine serum, $1 \%$ nonessential amino acids, $4.5 \mathrm{~g} / \mathrm{L}$ glucose, $2 \mathrm{mmol} / \mathrm{L}$ L-glutamine, $100 \mathrm{U} / \mathrm{mL}$ penicillin, and $100 \mu \mathrm{g} / \mathrm{mL}$ streptomycin. Cells were seeded at a density of $6.25 \times 10^{4}$ cells $/ \mathrm{cm}^{2}$ onto 12-well transwell culture plates coated with polycarbonate filters with a pore size of $0.4 \mu \mathrm{m}$ and an area of $1.13 \mathrm{~cm}^{2}$ (Corning Incorporated, Corning, NY, USA). Growth media were added to the apical (AP) side and basolateral (BL) side. The medium was changed every other day. Cells were grown 21-28 days prior to use. The tightness of the cell monolayer was evaluated through the determination of trans-epithelial electrical resistance (TEER), which was measured using a Millicell-ERS Resistance Instrument (EMD Millipore, Billerica, MA, USA), and the permeation ratio of ${ }^{14} \mathrm{C}$ mannitol was determined using a liquid scintillation counter (Wallac MicroBeta TriLux; Wallac, Turku, Finland) during the transport experiment. The cell monolayer was applied for transport experiments once the TEER was $>600 \Omega / \mathrm{cm}^{2}$.

Confluent cultures of Caco-2 cell monolayers (cultured for 21 days) in Hank's buffered salt solution (HBSS) were precultured at $37^{\circ} \mathrm{C}$ for 20 minutes in an incubator. After removing the buffer solution, the cell monolayers were washed three times with HBSS. The experiments were performed at $37^{\circ} \mathrm{C}$ for 150 minutes from the $\mathrm{AP}$ to $\mathrm{BL}(\mathrm{A} \rightarrow \mathrm{B})$ or the $\mathrm{BL}$ to $\mathrm{AP}(\mathrm{B} \rightarrow \mathrm{A})$ directions. The donor chambers were filled with $1.5 \mathrm{~mL}$ of a sample solution containing $5 \mathrm{FU}(200 \mu \mathrm{M})$, and the receiver compartments were filled with $2.6 \mathrm{~mL}$ of blank HBSS, respectively. Samples were taken at receiver side $(100 \mu \mathrm{L})$ at different time points (30 minutes, 60 minutes, 90 minutes, 120 minutes, and 150 minutes), and the same volume of fresh HBSS was added immediately. At the end of the transport study, cells were recovered and washed three times with $2 \mathrm{~mL}$ of ice-cold $\mathrm{HBSS} ; 800 \mu \mathrm{L}$ of $\mathrm{NaOH}(0.2 \mathrm{~N})$ was then added to lyse the cells, and the sample was back neutralized by adding $200 \mu \mathrm{L}$ of $\mathrm{HCl}(0.8 \mathrm{~N})$. The amount of drug accumulated in the cell was evaluated. Each treatment was accomplished with 3-5 wells, and the experiments were conducted in duplicate. The 5FU content was determined as described earlier. The apparent permeability coefficient $\left(P_{\text {app }}, \mathrm{cm} / \mathrm{s}\right)$ for $5 \mathrm{FU}$ was calculated according to the following equation:

$$
P_{\text {app }}=\left(\frac{1}{A C_{0}}\right)\left(\frac{\mathrm{d} Q}{\mathrm{~d} t}\right)
$$

where $\mathrm{d} Q / \mathrm{d} t$ is the amount of drug accumulated in the receiver side during the interval time; $A$ is the surface area of the transwell membrane $\left(1.13 \mathrm{~cm}^{2}\right)$, and $C_{0}$ is the original donor concentration of $5 \mathrm{FU}$.

\section{Measurement of rectal retention time}

The method described by Choi et al ${ }^{30}$ was adopted for this study with slight modification. Male BALB/c mice ( $20 \mathrm{~g})$ were fasted and allowed free access to water for 24 hours prior to the experiments. 5FU microemulsion and TG5FU-ME with 0.1\% Cy7NHS ester (Seebio Biotech Co., Ltd., Shanghai, People's Republic of China) in the water phase were administered ( $20 \mathrm{mg} \mathrm{kg}^{-1}$ of $\left.5 \mathrm{FU}\right)$ into the rectum $0.5 \mathrm{~cm}$ above the anus using a stomach probe needle. The formulations retained in the rectum were detected after administration using small animal imaging technology.

\section{$5 \mathrm{FU}$ distribution in vivo}

As described in other reports, ${ }^{17,23,24}$ male Sprague Dawley rats $(\sim 300 \mathrm{~g})$ were used for this study. A total of 58 rats were randomly divided into three groups $(20,18$, and 20$)$, fasted, and allowed free access to water for 24 hours prior to drug administration. 5FU microemulsion, TG-5FU-ME, and $5 \mathrm{FU}$ thermosensitive gel ( $30 \mathrm{mg} \mathrm{kg}$ of $5 \mathrm{FU})$ were administered into the rectum using a stomach sonde needle fit into a plastic syringe. The 5FU dose used in the test was approximately three times higher than that of the human dose $\left(10 \mathrm{mg} \mathrm{kg}^{-1}\right)$.

The method of Galandiuk et $\mathrm{al}^{16}$ was used in this investigation. At 0.5 hours, 1 hour, 3 hours, 6 hours, 9 hours, and 12 hours after drug administration, three animals from each group were anesthetized. Cardiac puncture was performed to collect systemic blood, and the rats were sacrificed with exsanguinations. Rectal tissues and regional lymph nodes were harvested to determine the 5FU tissue concentrations. Serum and tissue samples were stored at $-70^{\circ} \mathrm{C}$ until analysis. All the surgeries were performed under sodium pentobarbital anesthesia, and all efforts were made to minimize the suffering.

The tissue and plasma samples were treated as described in our previous work. ${ }^{13}$ Briefly, the tissue sample was mashed and ground into a homogenate by adding $2 \mathrm{~mL}$ of distilled water. After standing for 30 minutes, the precipitate was removed by centrifugation, washed, and filtered with $0.4 \mathrm{~mL}$ purified water three times. Subsequently, $1 \mathrm{~mL}$ of $0.5 \mathrm{~mol} \mathrm{~L}^{-1}$ ammonium sulfate was added to precipitate proteins. 5FU in the supernatant was extracted with addition of ethyl acetate for three times with $0.5 \mathrm{~mL}$ of ethyl acetate each. The solution was vortexed for 0.5 minutes each time after ethyl acetate was 
added. The ethyl acetate layer was collected and centrifuged at 4,000 rpm for 5 minutes. The plasma samples were treated with protein precipitation and drug extraction. An accurately measured $1 \mathrm{~mL}$ sample of supernatant was removed and added into an Eppendorf tube, followed by dryness over nitrogen gas in a water bath at $40^{\circ} \mathrm{C}$. Then, the residue was dissolved in $100 \mu \mathrm{L}$ of the mobile phase, $20 \mu \mathrm{L}$ of which was measured accurately for 5FU concentration by HPLC (as discussed in the "Entrapment efficiency test" section).

\section{Rectal irritation}

Immediately after the last sampling, two animals from the TG-5FU-ME or 5FU thermosensitive gel group were collected, respectively. The rectum was isolated, washed with saline solution, fixed in 10\% neutral carbonate-buffered formaldehyde, embedded in paraffin, and cut into slices. The tissues were stained with hematoxylin-eosin and examined under light microscopy.

All animal studies were carried out in accordance with the recommendations in the Guide for the Care and Use of Laboratory Animals of the National Institutes of Health. The protocol was approved by the Committee of the Ethics of Animal Experiments of the Chinese Academy of Medical Sciences and Peking Union Medical College.

\section{Statistics}

The data were analyzed using the SPSS (11.5) software (SPSS Inc., Chicago, IL, USA). After validation of the test for homogeneity of variance, differences between or among study groups were examined by Student's $t$-test or by oneway analysis of variance followed by the Newman-Keuls test for multiple comparisons. The $P$-value $<0.05$ was considered statistically significant.

\section{Results and discussion Evaluation of 5FU microemulsion}

All the 5FU microemulsion vesicles were in round shape and free of aggregation, as assessed by the transmission electron microscope (Figure 1). Sample characteristics are given in Table 1 . The average size of 5FU microemulsion was $2.43 \pm 0.49 \mu \mathrm{m}$, with a narrow size distribution. Zeta potential value was $+1.06 \pm 0.11$. Encapsulation efficiency was $80.11 \% \pm 0.58 \%$.

\section{Physicochemical properties of TG-5FU-ME}

The physicochemical properties of drug-free thermosensitive gel and TG-5FU-ME, such as gel temperature, gel strength, coverage area, and bioadhesive force, are shown in Table 2.

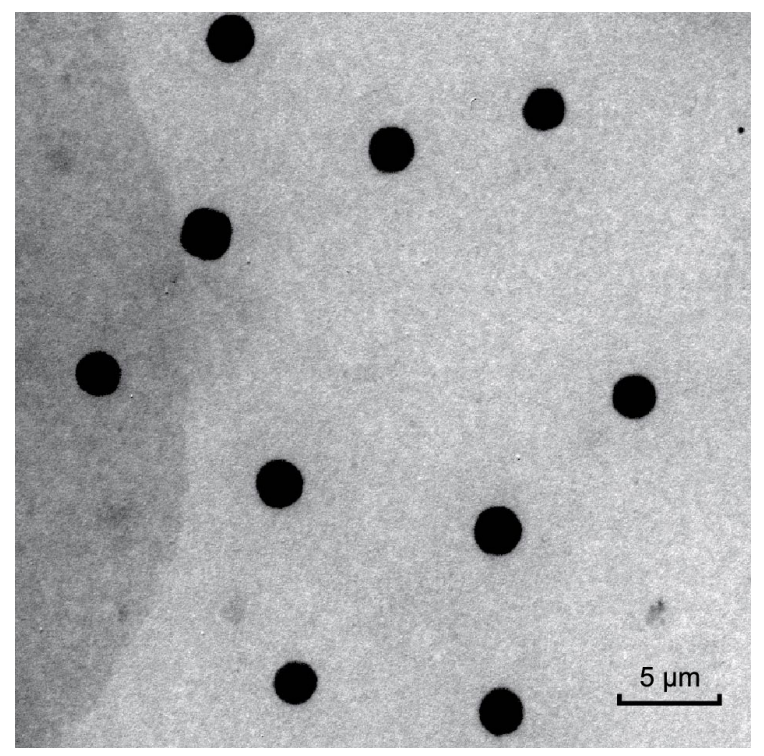

Figure I 5FU microemulsion image in TEM.

Note: The 5FU microemulsion particles were visualized under TEM for morphological feature and size distribution $(\times 6,000)$.

Abbreviations: 5FU, 5-fluorouracil; TEM, transmission electron microscopy.

Addition of 5FU microemulsion into the thermosensitive gel decreased the gelling temperature from $54.5^{\circ} \mathrm{C}$ to $19.2^{\circ} \mathrm{C}$, and reduced the coverage area from $1.42 \mathrm{~cm}^{2} / \mathrm{mL}$ to $0.81 \mathrm{~cm}^{2} / \mathrm{mL}$. However, the bioadhesive force and gel strength of thermosensitive gel were dramatically increased from $1.47 \mathrm{~N}$ to $4.18 \mathrm{~N}$ and $11.21 \mathrm{~g}$ to $30.14 \mathrm{~g}$, respectively. The TG-5FU-ME was designed for gelling at ambient temperature, turning into liquid form within 10 minutes below $10^{\circ} \mathrm{C}$ and returning to gel form at body temperature. Most likely, after applying as liquid forms to the rectum, a layer of gel containing 5FU microemulsion forms and spreads broadly on rectal mucous membrane, with reasonable gel strength and bioadhesive force, which might benefit the tissue deposition of drug and improve the patient compliance.

\section{Formulation stability study}

The results of naked eye evaluation, microscopic examination, and 5FU content test of 5FU microemulsion and TG-5FU-ME are shown in Figures 2 and 3, as well as in Tables 3 and 4. The 5FU microemulsion was unstable under the temperature of $20^{\circ} \mathrm{C} \pm 1^{\circ} \mathrm{C}$, with layer occurring in 24 hours after preparation, and the oil precipitation took place in 1 week (Figure 2A-D). Microscopically, the droplet of microemulsion began to merge in 24 hours and became large bulbs in 1 week (Figure 2E-H) with the disappearance of the microemulsion droplet structure. In contrast, TG-5FU-ME was remarkably stable for the period of study (4 months) at ambient temperature. This system gelled at room temperature (Figure $3 \mathrm{~A}-\mathrm{E}$ ) and turned into liquid form (Figure $3 \mathrm{~A}^{\prime}-\mathrm{E}^{\prime}$ ) 
Table I Characteristics of 5FU microemulsion

\begin{tabular}{llllll}
\hline Mean diameter $(\mu \mathrm{m})$ & Viscosity $(\mathrm{cP})$ & Variance $(\mathrm{PDI})$ & Drug content $(\% \mathrm{w} / \mathrm{v})$ & Encapsulation efficiency $(\%)$ & Zeta potential \\
\hline $2.12 \pm 0.33$ & $9.1 \mathrm{I} \pm 1.03$ & $0.2 \mathrm{I} \pm 0.01 \mathrm{I}$ & $4.07 \pm 0.12$ & $84.23 \pm 0.62$ & $+2.0 \mathrm{I} \pm 0.1 \mathrm{I}$ \\
\hline
\end{tabular}

Note: Values are presented as mean \pm SD of three independent experiments.

Abbreviations: 5FU, 5-fluorouracil; PDI, polydispersity index; SD, standard deviation.

Table 2 Physicochemical properties of the drug-free thermosensitive gel and TG-5FU-ME

\begin{tabular}{lllll}
\hline Samples & Gel temperature $\left({ }^{\circ} \mathbf{C}\right)$ & Gel strength $(\mathbf{g})$ & Coverage area $\left(\mathrm{cm}^{2} / \mathbf{m L}\right)$ & Bioadhesive force $(\mathbf{N})$ \\
\hline Thermosensitive gel & $54.5 \pm 2.7$ & $11.21 \pm 2.27$ & $1.42 \pm 0.13$ & $1.14 \pm 0.13$ \\
TG-5FU-ME & $19.2 \pm 1.2$ & $30.14 \pm 2.54$ & $0.81 \pm 0.11$ & $4.18 \pm 0.37$ \\
\hline
\end{tabular}

Note: Values are presented as mean \pm SD of three independent experiments.

Abbreviations: TG-5FU-ME, thermosensitive gel-mediated 5FU water-in-oil microemulsion; SD, standard deviation.
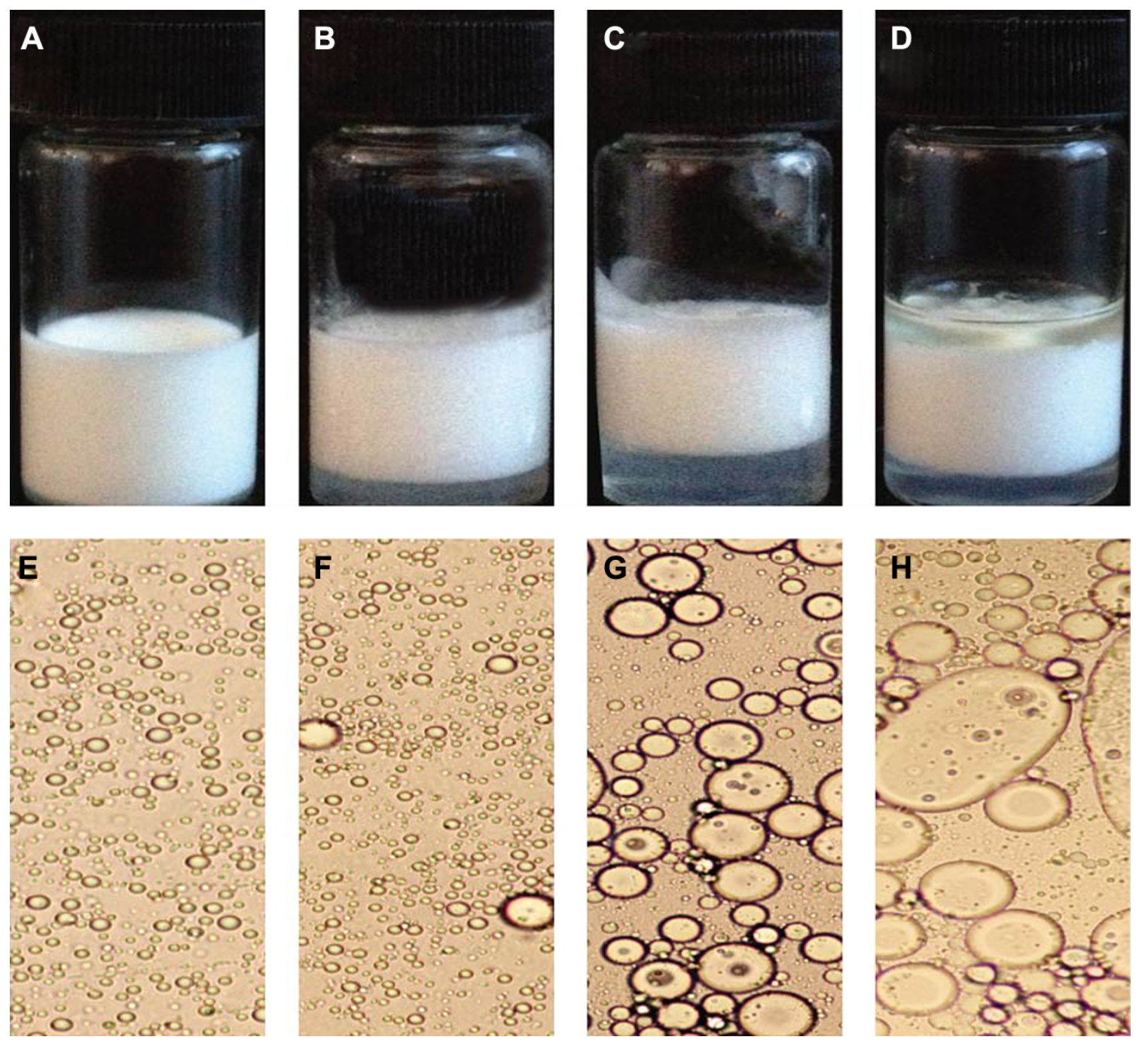

Figure 2 Appearance of 5FU microemulsion

Notes: $5 \mathrm{FU}$ microemulsion stored at $20^{\circ} \mathrm{C} \pm \mathrm{I}^{\circ} \mathrm{C}$ was examined right after the preparation (A, E), as well as after storage for 24 hours (B, F), 72 hours (C, G), and I week (D, H). (A-D) Naked eye examination; (E-H) microscopic examination.

Abbreviation: 5FU, 5-fluorouracil. 

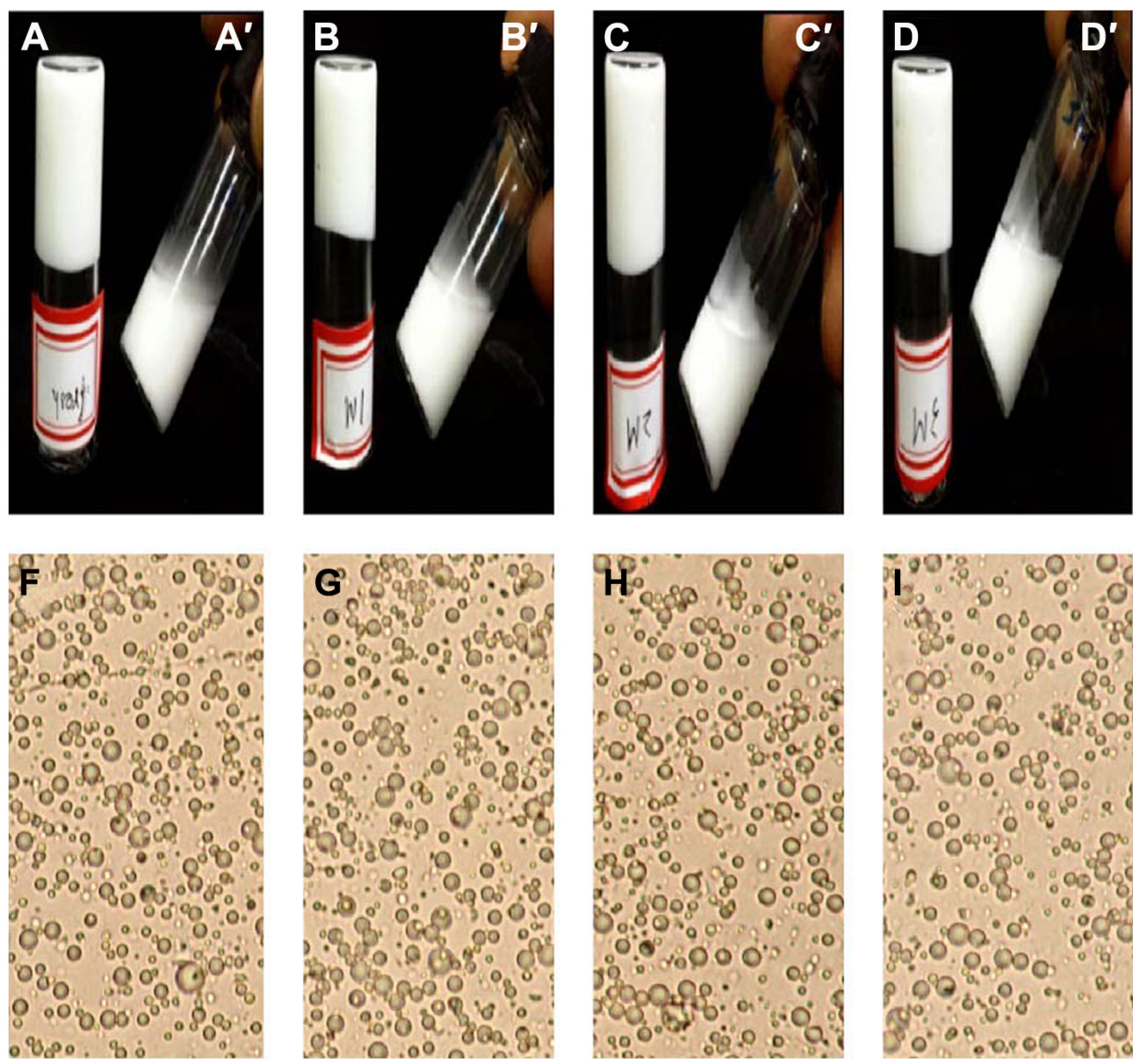
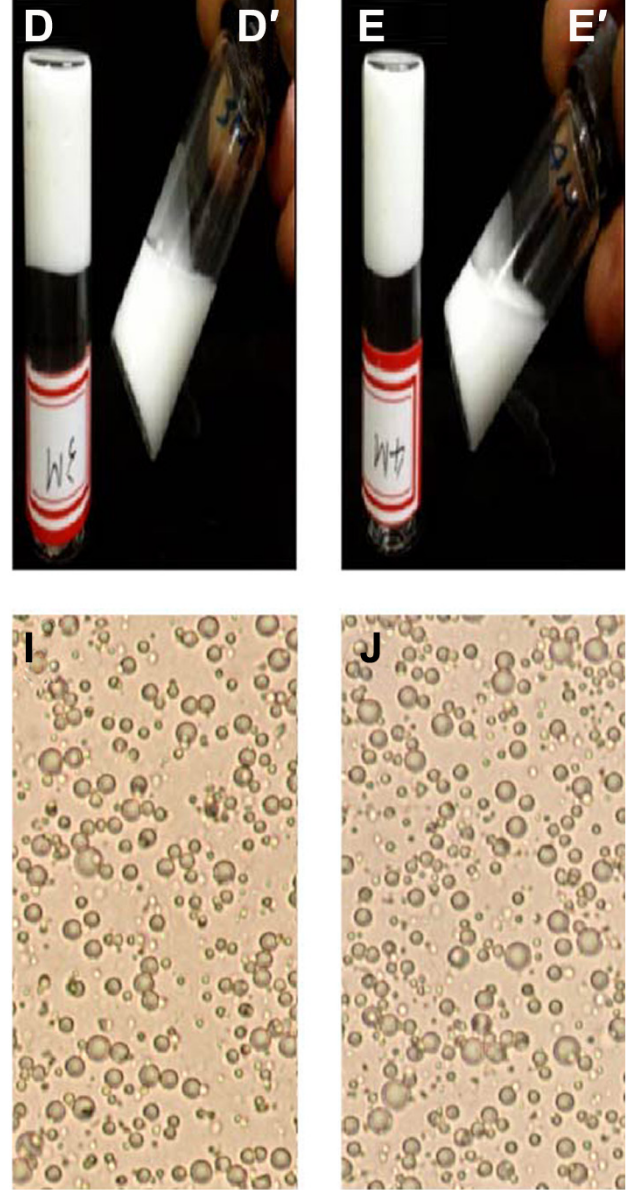

Figure 3 Appearances of TG-5FU-ME.

Notes: Freshly prepared TG-5FU-ME samples were placed at $20^{\circ} \mathrm{C} \pm \mathrm{I}^{\circ} \mathrm{C}$ for 0 hour (A, $\left.\mathbf{A}^{\prime}, \mathbf{F}\right), \mathbf{I}$ month (B, $\left.\mathbf{B}^{\prime}, \mathbf{G}\right), 2$ months $\left(\mathbf{C}, \mathbf{C}^{\prime}, \mathbf{H}\right), 3$ months $\left(\mathbf{D}, \mathbf{D}^{\prime}, \mathbf{I}\right)$, and 4 months (E, $\left.\mathbf{E}^{\prime}, \mathbf{J}\right)$, followed by naked eye and microscopic examination. (A-E) Naked eye examination for the solid form of TG-5FU-ME at RT; (A'-E') naked eye examination for the liquid form sample at temperature below $10^{\circ} \mathrm{C}$; $(\mathbf{F}-\mathbf{J})$ microscopic examination. 1000× magnification.

Abbreviations: RT, room temperature; TG-5FU-ME, thermosensitive gel-mediated 5FU water-in-oil microemulsion.

Table 3 Stability of 5FU microemulsion

\begin{tabular}{lllll}
\hline Examination items & Freshly prepared & $\mathbf{2 4}$ hours & $\mathbf{7 2}$ hours & I week \\
\hline Diameters $(\mu \mathrm{m})$ & $2.67 \pm 0.3 \mathrm{I}^{\mathrm{a}}$ & $10.22 \pm 1.27$ & $20.14 \pm 1.73$ & ND \\
In visual appearance & Homogeneous & Layering & Layering & Layering, oil precipitation \\
5FU content $(\%)$ & $99.24 \pm 1.31$ & $101.04 \pm 2.21$ & $99.27 \pm 1.54$ & $100.68 \pm 1.17$ \\
\hline
\end{tabular}

Note: a Values are presented as mean \pm SD of three independent experiments.

Abbreviations: 5FU, 5-fluorouracil; ND, not detectable; SD, standard deviation.

Table 4 Stability of TG-5FU-ME

\begin{tabular}{llllll}
\hline Examination items & Freshly prepared & I month & 2 months & 3 months & 4 months \\
\hline Diameters $(\mu \mathrm{m})$ & $2.51 \pm 0.49^{\mathrm{a}}$ & $2.56 \pm 0.60$ & $2.61 \pm 0.37$ & $2.73 \pm 0.73$ & $2.67 \pm 0.57$ \\
In visual appearance & Homogeneous & Homogeneous & Homogeneous & Homogeneous & Homogeneous \\
$5 \mathrm{FU}$ content $(\%)$ & $101.35 \pm 1.1 \mathrm{II}$ & $99.24 \pm 2.04$ & $98.91 \pm 2.34$ & $99.69 \pm 1.08$ & $99.83 \pm 2.04$ \\
\hline
\end{tabular}

Note: ${ }^{\vee}$ alues are presented as mean \pm SD of three independent experiments.

Abbreviations: 5FU, 5-fluorouracil; TG-5FU-ME, thermosensitive gel-mediated 5FU water-in-oil microemulsion; SD, standard deviation. 
within 10 minutes when the temperature was below $10^{\circ} \mathrm{C}$. The sign of instability, such as layering, creaming, coalescence, phase separation, and precipitation, was not detected; significant morphological changes were detected negative as well (Figure 3F-J). The 5FU content did not change during the storage time in both the 5FU microemulsion and TG5FU-ME. A heating-cooling cycle test was also conducted, and the results were in consistent with that of the formulation stability study (data not shown). The improved stability of this system might be related to the protective effect of the gel in its action against droplet merge and phase transfer of the 5FU microemulsion.

\section{In vitro release}

The in vitro release profiles of the different formulations are shown in Figure 4. The 5FU solution was completely released within 30 minutes, and 5FU thermosensitive gel released within 2 hours. The 5FU microemulsion prolonged the release to 8 hours, and TG-5FU-ME increased the releasing time to 10 hours.

It has been proven that microemulsion system possesses sustained drug release property due to the resistance rendered by the oil phase which blocks the diffusion of the drug from inner phase into the release medium. ${ }^{27}$ Furthermore, studies demonstrate that the drug release from thermosensitive gels follows the Higuchi square root law, and the diffusion coefficient of the drug in the gel declines with increasing levels of both poloxamers and bioadhesive material and is consistent with a consequent increase in bulk viscosity and gel rigidity. ${ }^{36-39} \mathrm{We}$ assume that when the microemulsion

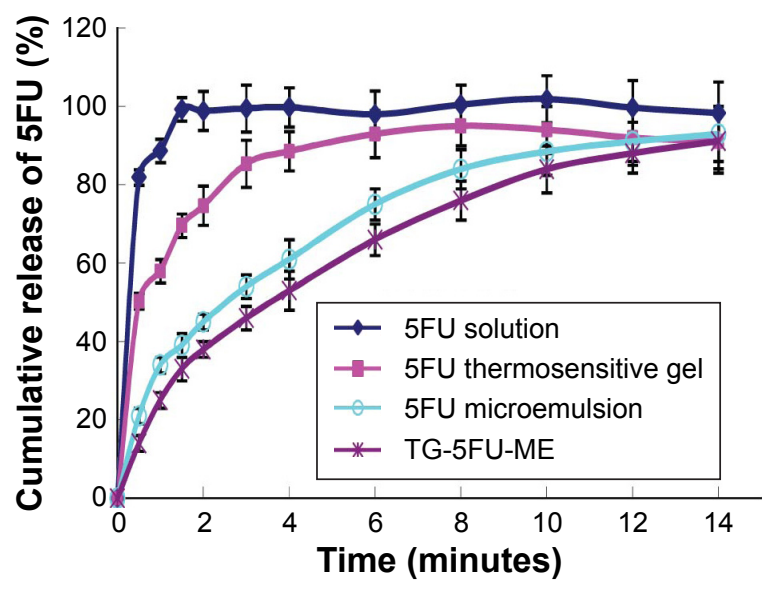

Figure 4 Release profiles of $5 F U$ formulations in vitro.

Notes: Release features of 5FU in 5FU solution, 5FU thermosensitive gel, 5FU microemulsion, and TG-5FU-ME were measured in a dynamic dialysis assay.

Abbreviations: 5FU, 5-fluorouracil; TG-5FU-ME, thermosensitive gel-mediated 5FU water-in-oil microemulsion. system was entrapped in the thermosensitive gel, both of those systems would influence the releasing character of the drug. Further studies are needed to clarify the releasing properties and mechanisms of the microemulsion entrapped thermosensitive gel system.

\section{Trans Caco- 2 cell test}

TEER measurements and mannitol transport were used to test cellular integrity. The results showed that there was no significant variation in the TEER values for all the samples in the test, and the average transport of mannitol $\left(0.91 \pm 0.06 \times 10^{-6} \mathrm{~cm} / \mathrm{s}\right)$ did not vary significantly after exposure to $5 \mathrm{FU}$ thermosensitive gel $\left(0.88 \pm 0.03 \times 10^{-6} \mathrm{~cm} / \mathrm{s}\right), 5 \mathrm{FU}$ microemulsion $\left(0.92 \pm 0.05 \times 10^{-6} \mathrm{~cm} / \mathrm{s}\right)$, and TG-5FU-ME $\left(0.94 \pm 0.07 \times 10^{-6} \mathrm{~cm} / \mathrm{s}\right)$, indicating that the cell monolayers remained intact after treatment.

The apparent permeability coefficient $\left(P_{\text {app }}\right)$ evaluates the velocity of a drug crossing the cell monolayer. ${ }^{40}$ As shown in Figure 5, for 5FU thermosensitive gel, 5FU microemulsion, or TG-5FU-ME, the amount of 5FU transported from $\mathrm{A} \rightarrow \mathrm{B}$ or from $\mathrm{B} \rightarrow \mathrm{A}$ across the Caco- 2 monolayer was in an identical pattern, which was proportional to time in 150 minutes, with no significant difference of the $P_{\text {app }}$ value between the $\mathrm{A} \rightarrow \mathrm{B}$ and $\mathrm{B} \rightarrow \mathrm{A}$. The $P_{\text {app }}$ values of $5 \mathrm{FU}$ microemulsion and TG-5FU-ME were $1.24 \times 10^{-5} \mathrm{~cm} / \mathrm{s}$ and $1.25 \times 10^{-5} \mathrm{~cm} / \mathrm{s}$, respectively, approximately six times higher than that of $5 \mathrm{FU}$ thermosensitive gel $\left(1.99 \times 10^{-6} \mathrm{~cm} / \mathrm{s}\right.$; as shown in Figure 6). Furthermore, in the $\mathrm{A} \rightarrow \mathrm{B}$ direction, 5FU intracellular accumulation of the 5FU microemulsion and TG-5FU-ME was $17.45 \%$ and $18.41 \%$ of the given amount, respectively, which were greater than that of the 5FU thermosensitive gel $(3.41 \%$; as shown in Figure 7). As the cell monolayers remained intact after treatment as described earlier, the increased intracellular accumulation and transportation of $5 \mathrm{FU}$ for $5 \mathrm{FU}$ microemulsion or TG-5FU-ME appeared to be attributable to the improved lipophilicity of the formulation.

$5 \mathrm{FU}$ is a hydrophilic drug and crosses the rectal epithelium primarily via passive transport through the water-filled pores. Tight junction between cells greatly restricts the transport and consequently reduces the absorption of drug. The introduction of a microemulsion system increased the lipophilicity of the formulation which facilitated passive diffusion or/and clathrin-mediated endocytosis. ${ }^{41}$

\section{Measurement of rectal retention time}

5FU microemulsion and TG-5FU-ME with $0.1 \%$ Cy7NHS ester added in the water phase were administered (20 $\mathrm{mg} \mathrm{kg}^{-1}$ of $5 \mathrm{FU}$ ) into the rectum of mouse $0.5 \mathrm{~cm}$ 

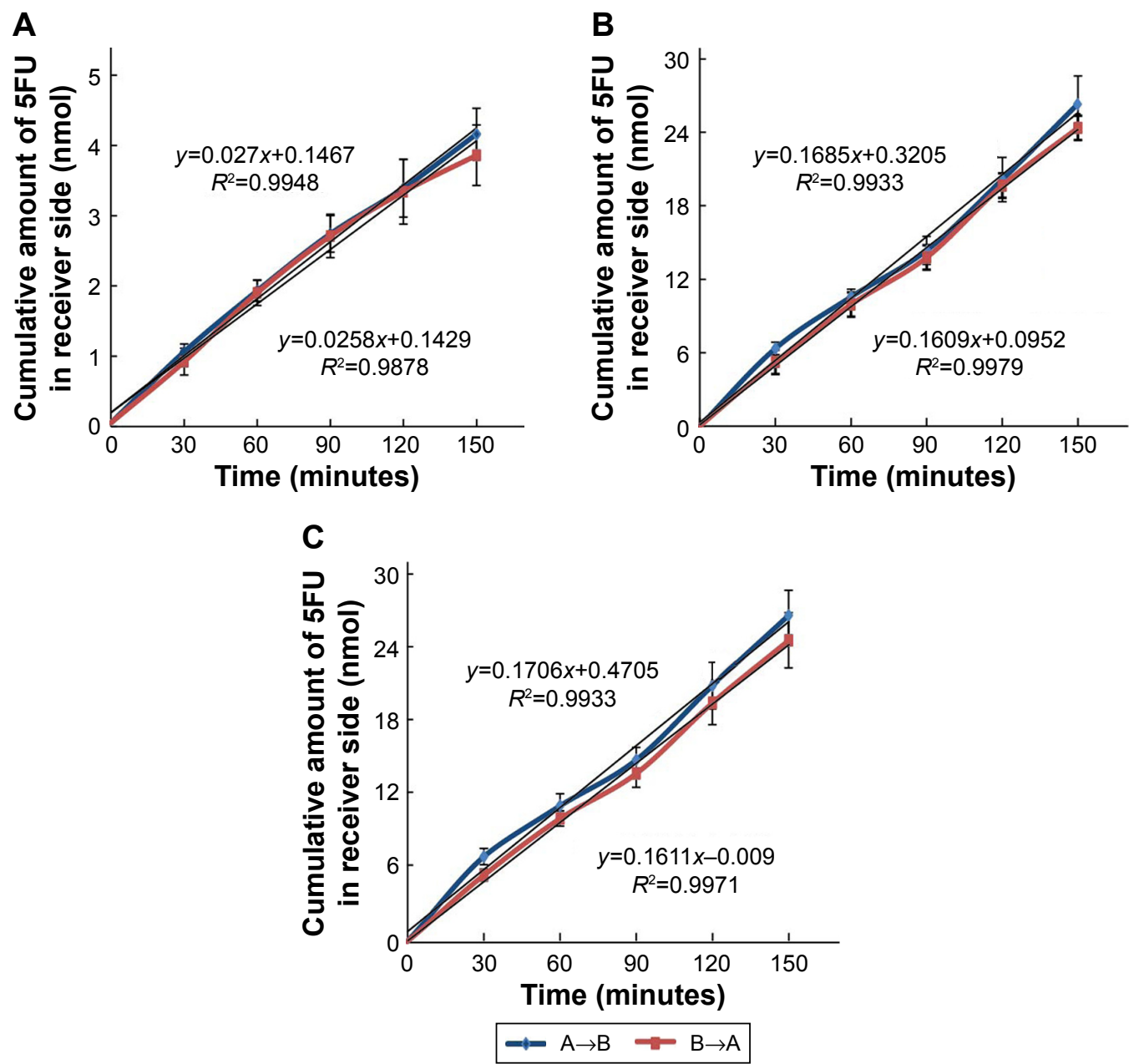

Figure $5 \mathrm{AP}$ to $\mathrm{BL}(\mathrm{A} \rightarrow \mathrm{B})$ and $\mathrm{BL}$ to $\mathrm{AP}(\mathrm{B} \rightarrow \mathrm{A})$ transport of $5 \mathrm{FU}$.

Notes: Experiments were performed at $37^{\circ} \mathrm{C}$ for 150 minutes for both directions. The donor chambers were filled with I.5 mL of a sample solution containing $5 \mathrm{FU}$ ( $200 \mu \mathrm{M}$; mean $\pm \mathrm{SD}, \mathrm{n}=3$ ). A represents $5 \mathrm{FU}$ solution; $B$ represents $5 \mathrm{FU}$ micro emulsion; $\mathrm{C}$ represents TG-5FU-ME.

Abbreviations: AP, apical; BL, basolateral; 5FU, 5-fluorouracil; SD, standard deviation.

above the anus using a stomach probe needle. As shown in Figure 8, the 5FU microemulsion flew out within 10 minutes after administration, while TG-5FU-ME sustained at the applied site for $>5$ hours. This phenomenon suggested that the bioadhesive force and gel strength of TG-5FU-ME were sufficient to hold the formulation in the rectum for a time period much longer than that of the microemulsion.

\section{$5 F U$ distribution in vivo}

As compared to the 5FU thermosensitive gel group, the $5 \mathrm{FU}$ concentration in rectal tissue was significantly higher in the TG-5FU-ME group after 3 hours than that in the 5FU thermosensitive gel group (Figure 9A; $P<0.05$ ). This result is consistent with that achieved in the Caco- 2 transport study (Figure 7) and in vitro release study (Figure 4), and could be explained by the sustained drug release, increased rectal mucous membrane permeation, and enhanced intracellular

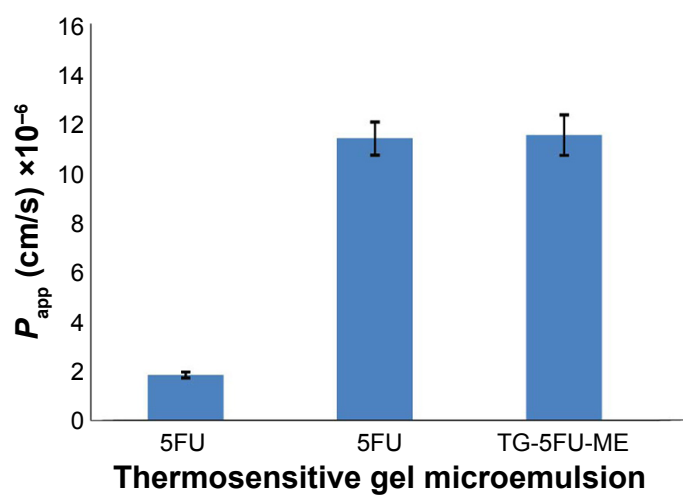

Figure 6 Formulation change affects transportation efficacy of 5FU crossing the Caco-2 cell monolayers.

Notes: The donor chambers were filled with $1.5 \mathrm{~mL}$ of a sample solution containing $5 \mathrm{FU}(200 \mu \mathrm{M})$, and the receiver compartments were filled with $2.6 \mathrm{~mL}$ of blank HBSS. Samples were taken at receiver side. The determination of $5 \mathrm{FU}$ in transport buffer was carried out by HPLC. The apparent permeability coefficient $\left(P_{\text {app }} ; \mathrm{cm} / \mathrm{s}\right)$ for $5 F U$ was calculated according to the equation: $P_{\text {app }}=\left(I / A C_{0}\right)(\mathrm{d} Q / \mathrm{dt}$; mean $\pm \mathrm{SD}, \mathrm{n}=3)$. Abbreviations: 5FU, 5-fluorouracil; HBSS, Hank's buffered salt solution; HPLC, high-performance liquid chromatography; TG-5FU-ME, thermosensitive gelmediated 5FU water-in-oil microemulsion; SD, standard deviation. 


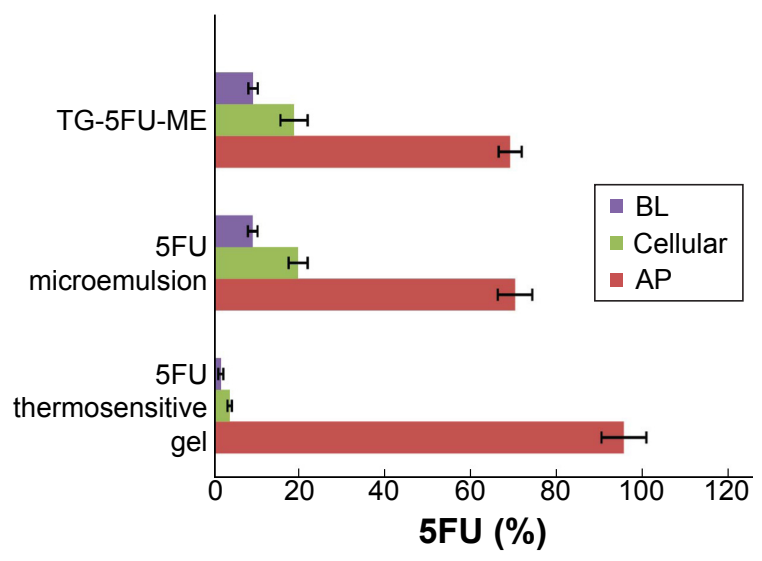

Figure 7 Absorption of $5 \mathrm{FU}$ in vitro.

Notes: Caco-2 cells were exposed to $5 \mathrm{FU}$ thermosensitive gel, $5 \mathrm{FU}$ microemulsion, or TG-5FU-ME (containing 5FU, $200 \mu \mathrm{M}$ ). The percentage of 5FU in AP and BL media and cell monolayer was determined in 2.5 hours (mean $\pm S D, n=3$ ).

Abbreviations: AP, apical; BL, basolateral; 5FU, 5-fluorouracil; TG-5FU-ME, thermosensitive gel-mediated 5FU water-in-oil microemulsion; SD, standard deviation.

uptake in TG-5FU-ME treatment. In the regional lymph nodes, the 5FU concentration in the TG-5FU-ME group and $5 \mathrm{FU}$ microemulsion group was found to be significantly higher (Figure 9) than that in the 5FU thermosensitive gel group $(P<0.05$ for both), which agrees with the results of other researchers. ${ }^{17}$ It is worthy to note that the high rectal and regional lymph node concentrations lasted for up to 9 hours after TG-5FU-ME administration, whereas in the 5FU microemulsion group drug concentrations remained high only for 2 hours. TG-5FU-ME seemed to acquire the advantage of both the 5FU microemulsion and 5FU thermosensitive gel, showing an increased transportation and rectal retention time. Blood level of 5FU in the 5FU microemulsion group as well as the TG-5FU-ME group was low and showed no significant difference from that of the 5FU thermosensitive gel group ( $P>0.05$ for both), suggesting a reduced chance of causing side effect.

\section{Rectal irritation}

Rectal tissue morphological test showed that neither 5FU thermosensitive gel nor TG-5FU-ME caused irritation or damage on rectal tissues during the treatment course (Figure 10). This is at least partially due to their good spreading property, which leads to a relatively low drug concentration per unit square of the rectal mucous membrane. In addition, the encapsulation of the drug in the emulsion minimized the direct contact of the drug with the rectal mucous.
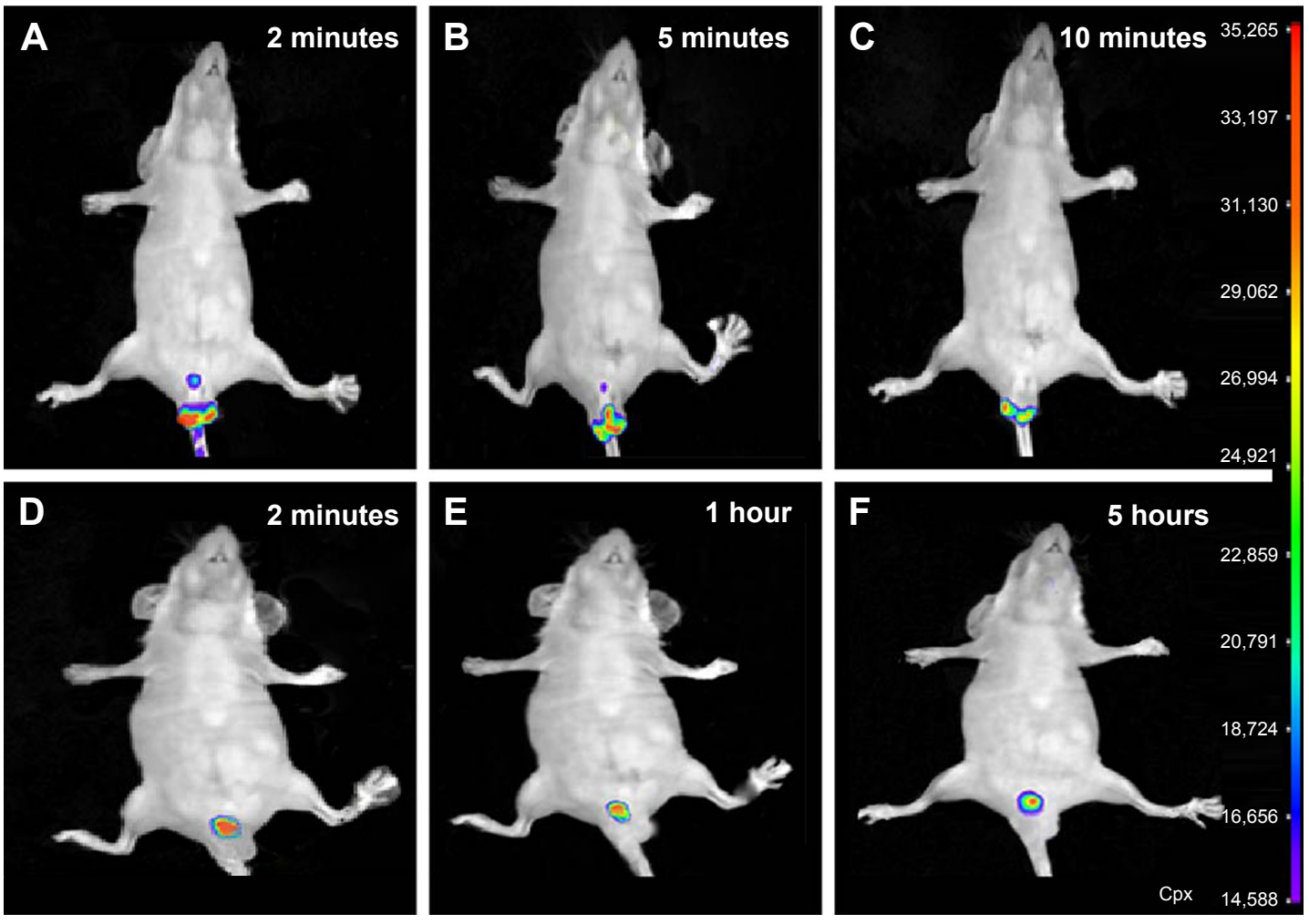

Figure 8 Rectal retention time.

Notes: 5FU microemulsion and TG-5FU-ME with $0.1 \%$ Cy7NHS ester were administered into the rectum, $0.5 \mathrm{~cm}$ above the anus using a stomach probe needle. (A-C) 5FU microemulsion (2 minutes, 5 minutes, 10 minutes after administration); (D-F) TG-5FU-ME (2 minutes, 2 hours, 5 hours after administration).

Abbreviations: 5FU, 5-fluorouracil; TG-5FU-ME, thermosensitive gel-mediated 5FU water-in-oil microemulsion; cpx, count per second. 
A

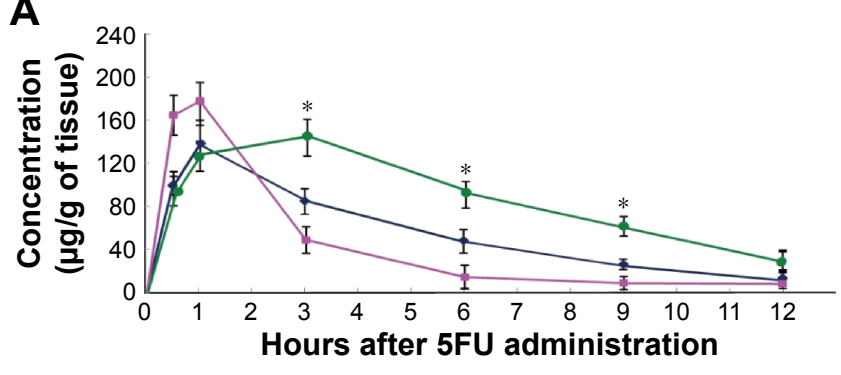

B

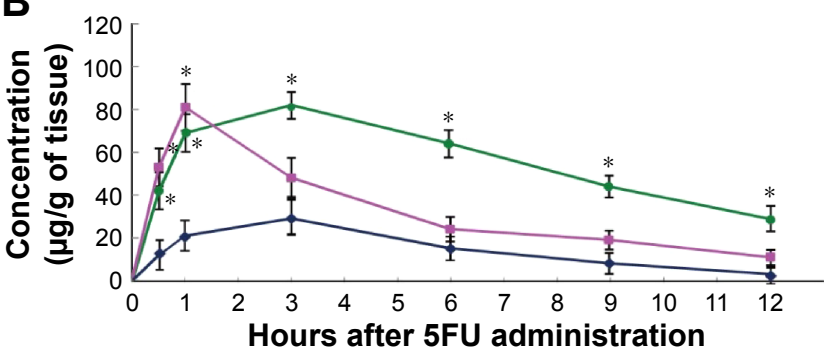

C

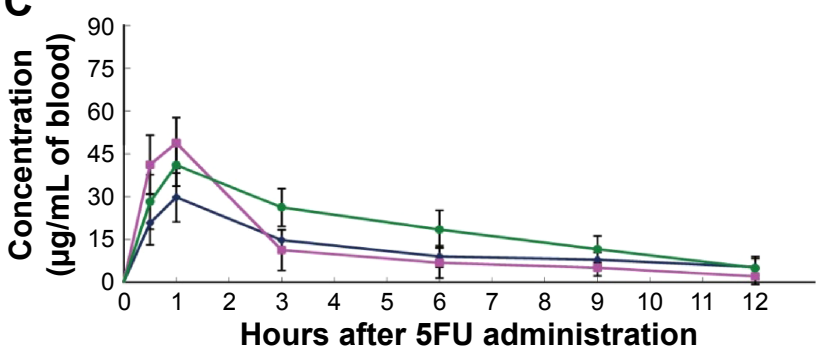

5FU thermosensitive gel $\rightarrow-5$ FU microemulsion $\rightarrow-$ TG-5FU-ME

Figure 9 Tissue distribution of 5FU.

Notes: 5FU concentrations in rectal tissue $(\mathbf{A})$, regional lymph node $(\mathbf{B})$, and systemic blood $(\mathbf{C})$ after rectal route administration of $20 \mathrm{mg} \mathrm{kg}^{-1} \mathrm{TG}-5 \mathrm{FU}-\mathrm{ME}(\rightarrow-)$, $5 \mathrm{FU}$ microemulsion (--), or 5FU thermosensitive gel $(-\rightarrow)$. $* P<0.05$ (TG-5FU-ME vs thermosensitive gel; mean $\pm S D, n=3$ ).

Abbreviations: 5FU, 5-fluorouracil; TG-5FU-ME, thermosensitive gel-mediated 5FU water-in-oil microemulsion; SD, standard deviation.

\section{Conclusion}

The TG-5FU-ME formulation was designed to improve the in situ delivery of 5FU for the treatment of colon cancer. In this study, we showed that the microemulsion facilitated $5 \mathrm{FU}$ transportation into target tissue and the thermosensitive gel increased the stability of the 5FU microemulsion as well as the retention time in rectal membrane. The formulation is in a free-flowing liquid form when the temperature is below $10^{\circ} \mathrm{C}$. After applying to the rectum, a gel layer containing 5FU microemulsion forms and spreads broadly on rectal mucous membrane with appropriate gel strength and bioadhesive force, which could improve the patient's compliance and ameliorate their alienation. For chemotherapy, this formulation might be able to efficiently prolong the drug's rectal
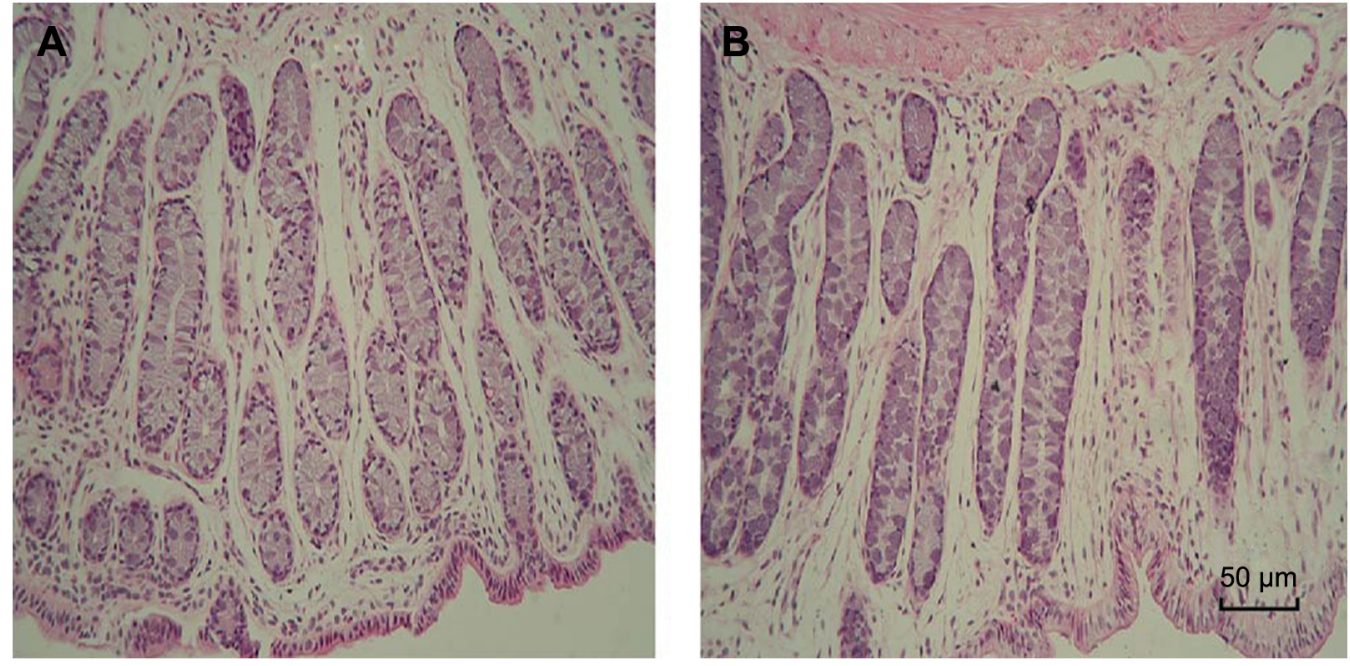

Figure 10 The morphology of rectal tissues after exposure to 5FU thermosensitive gel and TG-5FU-ME.

Notes: The rat's rectal tissue was taken 12 hours after TG-5FU-ME (A) and 5FU thermosensitive gel (B) administration (20 mg kg ${ }^{-1}$ of $\left.5 F U\right)$ through rectal route. Tissue slides were examined with $\mathrm{H} \& \mathrm{E}$ staining $(\times 200)$.

Abbreviations: 5FU, 5-fluorouracil; H\&E, hematoxylin and eosin; TG-5FU-ME, thermosensitive gel-mediated 5FU water-in-oil microemulsion. 
retention time, enlarge the drug's contact area with diseased tissue, and improve the drug's permeability. With regard to safety concern, it could reduce the direct contact of the drug with the rectum site. Our study validated the advantages of this formulation, and therefore, TG-5FU-ME could be considered a promising rectal delivery system for the treatment of rectal cancer. Furthermore, this dosage form would be a promising alternative applied in other mucosal drug delivery systems such as ocular, nasal, buccal, and vaginal.

\section{Author contributions}

All authors contributed toward data analysis, drafting and critically revising the paper and agree to be accountable for all aspects of the work.

\section{Disclosure}

The authors report no conflicts of interest in this work.

\section{References}

1. Allemani C, Weir HK, Carreira H, et al. Global surveillance of cancer survival 1995-2009: analysis of individual data for 25,676,887 patients from 279 population-based registries in 67 countries (CONCORD-2). Lancet. 2015;385(9972):977-1010.

2. Torre LA, Bray F, Siegel RL, Ferlay J, Lortet-Tieulent J, Jemal A. Global Cancer Statistics, 2012. CA Cancer J Clin. 2015;65(2):87-108.

3. Siegel R, Ma J, Zou Z, Jemal A. Cancer Statistics, 2014. CA Cancer J Clin. 2014;64(1):9-29.

4. Phillips TA, Howell A, Grieve RJ, Welling PG. Pharmacokinetics of oral and intravenous fluorouracil in humans. J Pharm Sci. 1980;69(12): 1428-1431.

5. Fraile RJ, Baker LH, Buroker TR, Horwitz J, Vaitkevicius VK. Pharmacokinetics of 5-fluorouracil administered orally, by rapid intravenous and by slow infusion. Cancer Res. 1980;40(7):2223-2228.

6. Longley DB, Harkin DP, Johnston PG. 5-Fluorouracil: mechanisms of action and clinical strategies. Nat Rev Cancer. 2003;3(5):330-338.

7. Parker WB, Cheng YC. Metabolism and mechanism of action of 5-fluorouracil. Pharmacol Ther. 1990;48(3):381-395.

8. Gamelin EC, DanquechinDorval EM, Dumesnil YF, et al. Relationship between 5-fluorouracil (5-FU) dose intensity and therapeutic response in patients with advanced colorectal cancer receiving infusional therapy containing 5-FU. Cancer. 1996;77(3):441-451.

9. Di Paolo A, Danesi R, Falcone A, et al. Relationship between 5-fluorouracil disposition, toxicity and dihydropyrimidine dehydrogenase activity in cancer patients. Ann Oncol. 2001;12(9):1301-1306.

10. Kuropkat C, Griem K, Clark J, Rodriguez ER, Hutchinson J, Taylor SG. Severe cardiotoxicity during 5-fluorouracil chemotherapy - a case and literature report. Am J Clin Oncol. 1999;22(5):466-470.

11. Anitha A, Maya S, Sivaram AJ, Mony U, Jayakumar R. Combinatorial nanomedicines for colon cancer therapy. Wiley Interdiscip Rev Nanomed Nanobiotechnol. 2016;8(1):151-159.

12. Jain SK, Chaurasiya A, Gupta Y, et al. Development and characterization of 5-FU bearing ferritin appended solid lipid nanoparticles for tumour targeting. J Microencapsul. 2008;25(5):289-297.

13. Wang LL, Zheng WS, Chen SH, Fang XQ. Development of in situ gelling and bio adhesive 5-fluorouracil enema. PLoS One. 2013;8(8): e71037.

14. Pokorny RM, Wrightson WR, Lewis RK, et al. Suppository administration of chemotherapeutic drugs with concomitant radiation for rectal cancer. Dis Colon Rectum. 1997;40(12):1414-1420.
15. Takahashi T, Kohno K, Yamaguchi T, Narisawa T. Preoperative use of 5-fluorouracil suppository for carcinoma of the rectum. Am J Surg. 1982; 143(2):183-185.

16. Galandiuk S, Wrightson W, Marr L, Myers S, LaRocca RV. Suppository delivery of 5-fluorouracil in rectal cancer. Ann Surg Oncol. 1996; 3(3):270-276.

17. Ichikawa D, Yamaguchi T, Yoshioka YJ, Sawai K, Takahashi T. Prognostic evaluation of preoperative combined treatment for advanced cancer in the lower rectum with radiation, intraluminal hyperthermia, and 5-fluorouracil suppository. Am J Surg. 1996;171(3):346-350.

18. Horie H, Kashiwagi H, Konishi F, Furuta K, Ozawa A, Kanazawa K. Improved outcome following preoperative radiochemotherapy: $40.5 \mathrm{~Gy}$ accelerated hyperfractionation and 5-fluorouracil suppositories for patients with carcinoma of the lower rectum. Surg Today. 1999; 29(10):992-998.

19. Paek SH, Xuan JJ, Choi HG, et al. Poloxamer 188 and propylene glycolbased rectal suppository enhances anticancer effect of 5-fluorouracil in mice. Biol Pharm Bull. 2006;29(5):1060-1063.

20. Nishioka B, Watanabe S, Fijita Y, et al. Clinical studies of intrarectal administration of 5FU emulsion as an adjunct to surgical treatment for rectal cancer. Jpn J Surg. 1980;10(2):110-114.

21. Watanabe S, Nishioka B, Fujita Y, et al. Experimental studies of intrarectal administration of emulsified 5FU as an adjuvant to the surgical treatment of rectal cancer. Jpn J Surg. 1980;10(2):17-21.

22. Rougier P, Nordlinger B. Large-scale trial for adjuvant treatment in high-risk resected colorectal cancers - rationale to test the combination of loco-regional and systemic chemotherapy and to compare 1-leucovorin plus 5-FU to levamisole plus 5-FU. Ann Oncol. 1993;4(suppl 2): S21-S28.

23. Anitha A, Maya S, Chennazhi KP, Lakshmanan V, Jayakumar R. In vitro combinatorial anticancer effects of 5-fluorouracil and curcumin loaded N, O-carboxymethyl chitosan nanoparticles towards colon cancer and in vivo pharmacokinetic studies. Eur J Pharm Biopharm. 2014;88(1):238-251.

24. Anitha A, Deepa N, Chennazhi KP, Lakshmanan V, Jayakumar R. Combinatorial anticancer effects of curcumin and 5-fluorouracil loaded thiolated chitosan nanoparticles towards colon cancer treatment. Biochim Biophys Acta. 2014;1840(9):2730-2743.

25. Saeednia L, Usta A, Asmatulu R. Preparation and Characterization of Drug-loaded Thermosensitive Hydrogels. San Diego, CA: ASME International Mechanical Engineering Congress and Exposition; 2013:15-21.

26. Shishu, Kamalpreet, Maheshwari M. Development and evaluation of novel microemulsion based oral formulations of 5-fluorouracil using non-everted rat intestine sac model. Drug Dev Ind Pharm. 2012; 38(3):294-300.

27. Tenjarla S. Microemulsions: an overview and pharmaceutical applications. Crit Rev Ther Drug Carrier Syst. 1999;16(5):461-521.

28. Hauss DJ, Fogal SE, Ficorilli JV, et al. Lipid-based delivery systems for improving the bioavailability and lymphatic transport of a poorly water-soluble LTB4 inhibitor. J Pharm Sci. 1998;87(2):164-169.

29. Chen HB, Chang XL, Du DR, Li J, Xu HB, Yang XL. Microemulsionbased hydrogel formulation of ibuprofen for topical delivery. Int J Pharm. 2006;315(1-2):52-58.

30. Choi HG, Oh YK, Kim CK. In situ gelling and mucoadhesive liquid suppository containing acetaminophen: enhanced bioavailability. Int J Pharm. 1998;165(1):23-32.

31. Kim CK, Lee SW, Choi HG, et al. Trials of in situ gelling and mucoadhesive acetaminophen liquid suppository in human subjects. Int J Pharm. 1998;174(1-2):201-207.

32. Park YJ, Yong CS, Kim HM, et al. Effect of sodium chloride on the release, absorption and safety of diclofenac sodium delivered by poloxamer gel. Int J Pharm. 2003;263(1-2):105-111.

33. Ryu JM, Chung SJ, Lee MH, Kim CK, Shim CK. Increased bioavailability of propranolol in rats by retaining thermally gelling liquid suppositories in the rectum. J Control Release. 1999;59(2):163-172. 
34. Klimaszewska K, Bernier-Cardou M, Cyr DR, Sutton BCS. Influence of gelling agents on culture medium gel strength, water availability, tissue water potential, and maturation response in embryogenic cultures of Pinus strobus L. In Vitro Cell Dev Biol Plant. 2000;36(4):279-286.

35. Koffi AA, Agnely F, Ponchel G, Grossiord JL. Modulation of the rheological and mucoadhesive properties of thermosensitive poloxamerbased hydrogels intended for the rectal administration of quinine. Eur J Pharm Sci. 2006;27(4):328-335.

36. Koffi AA, Agnely F, Besnard M, Brou JK, Grossiord JL, Ponchel G. In vitro and in vivo characteristics of a thermogelling and bioadhesive delivery system intended for rectal administration of quinine in children. Eur J Pharm Biopharm. 2008;69(1):167-175.

37. Ganguly S, Dash AK. A novel in situ gel for sustained drug delivery and targeting. Int J Pharm. 2004;276(1-2):83-92.
38. Moore T, Croy S, Mallapragada S, Pandit N. Experimental investigation and mathematical modeling of Pluronic (R) F127 gel dissolution: drug release in stirred systems. J Control Release. 2000;67(2-3):191-202.

39. Anderson BC, Pandit NK, Mallapragada SK. Understanding drug release from poly(ethylene oxide)-b-poly(propylene oxide)-b-poly(ethylene oxide) gels. J Control Release. 2001;70(1-2):157-167.

40. Chen MJ, Cheng YM, Lai PH, Wu JF, Hsu YC. In vitro biocompatibility of thermally gelling liquid mucoadhesive loaded curcuminoids in colorectal cancer chemoprevention. Int J Colorectal Dis. 2012; 27(7):869-878.

41. Jeong B, Kim SW, Bae YH. Thermosensitive sol-gel reversible hydrogels. Adv Drug Deliv Rev. 2002;54(1):37-51.

\section{Publish your work in this journal}

Drug Design, Development and Therapy is an international, peerreviewed open-access journal that spans the spectrum of drug design and development through to clinical applications. Clinical outcomes, patient safety, and programs for the development and effective, safe, and sustained use of medicines are the features of the journal, which has also been accepted for indexing on PubMed Central. The manuscript management system is completely online and includes a very quick and fair peer-review system, which is all easy to use. Visit http://www.dovepress.com/testimonials.php to read real quotes from published authors.

Submit your manuscript here: http://www.dovepress.com/drug-design-development-and-therapy-journal 